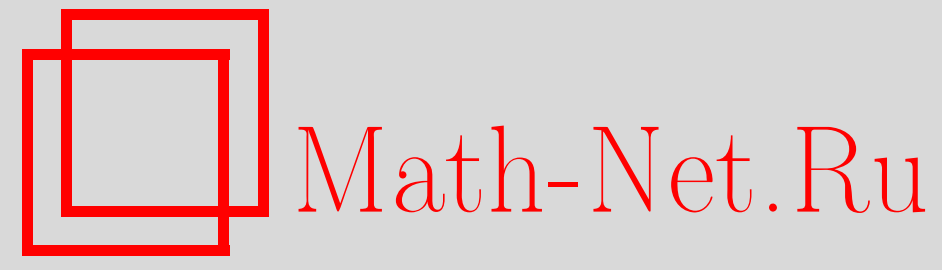

В. В. Сенатов, О построении асимптотических разложений высокой точности, Теория вероятн. и ее примен., 2015, том 60, выпуск 2, 290-310

DOI: https://doi.org/10.4213/tvp4620

Использование Общероссийского математического портала Math-Net.Ru подразумевает, что вы прочитали и согласны с пользовательским соглашением http: //www . mathnet.ru/rus/agreement

Параметры загрузки:

IP : 54.164 .48 .24

26 апреля 2023 г., 13:16:22

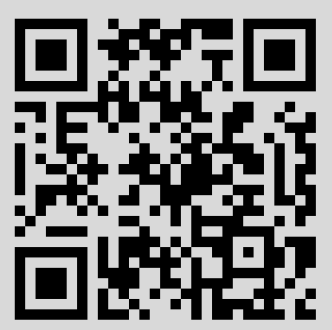




\title{
О ПОСТРОЕНИИ АСИМПТОТИЧЕСКИХ РАЗЛОЖЕНИЙ ВЫСОКОЙ ТОЧНОСТИ
}

\begin{abstract}
Предложены новые асимптотические разложения в центральной предельной теореме, позволяющие аппроксимировать распределения нормированных сумм независимых случайных величин с точностью, которая на несколько порядков превосходит точность оценки теоремы Берри-Эссеена.
\end{abstract}

Ключевые слова и фразы: асимптотические разложения, центральная предельная теорема, точность аппроксимации, теорема Берри-Эссеена.

Пусть $X_{1}, X_{2}, \ldots$ - независимые одинаково распределенные случайные величины с нулевым средним и единичной дисперсией. Пусть $P$ - общее распределение этих случайных величин, $P_{n}$ - распределение нормированной суммы $\left(X_{1}+\cdots+X_{n}\right) n^{-1 / 2}, \Phi-$ стандартный нормальный закон. В силу центральной предельной теоремы (ЦПТ) при больших $n$ распределение $P_{n}$ близко к $\Phi$. В связи с этим возникает задача об оценке точности аппроксимации распределений $P_{n}$ распределением $\Phi$ - одна из традиционных задач теории вероятностей. Далее нам понадобятся числовые характеристики распределения $P$, его моменты $\alpha_{k}=\int_{-\infty}^{\infty} x^{k} P(d x)$ и абсолютные моменты $\beta_{k}=\int_{-\infty}^{\infty}|x|^{k} P(d x)$, $k=1,2, \ldots$, по определению, $\alpha_{0}=\beta_{0}=1$. Говоря о моментах $k$-го порядка, мы всегда предполагаем, что они существуют.

Самым известным утверждением о точности аппроксимации в ЦПТ является теорема Берри-Эссеена, которая гарантирует, что при всех действительных $x$

$$
\left|F_{n}(x)-G(x)\right| \leqslant c \frac{\beta_{3}}{\sqrt{n}},
$$

где $F_{n}$ - функция распределения меры $P_{n}, G$ - функция распределения стандартного нормального закона, $c$ - постоянная. Задача о верхней оценке привлекала внимание десятков исследователей на протяжении последних семидесяти лет и недавно она получила решение, близкое к

*Московский государственный университет им. М. В. Ломоносова, механикоматематический факультет, Москва, Россия; e-mail: v.senatov@yandex.ru 
окончательному (см. [6]). Нижняя оценка $c$,

$$
c \geqslant \frac{3+\sqrt{10}}{6 \sqrt{2 \pi}}=0.4097 \ldots,
$$

была получена К.-Г. Эссееном в 1956 г. Так как в силу неравенства Ляпунова $\beta_{3} \geqslant 1$ для любого распределения $P$ с нулевым средним и единичной дисперсией, из нижней оценки $c$ следует, что теорема Берри-Эссеена может гарантировать точность 0.001 аппроксимации $F_{n}$ функцией $G$ лишь при $n>160000$, при $n$ порядка нескольких десятков утверждение теоремы Берри-Эссеена практически бессодержательно. Этот давно и хорошо известный факт привел к развитию нескольких направлений в исследовании точности аппроксимации в ЦПТ, среди которых - изучение асимптотических разложений. Справедливости ради стоит отметить, что асимптотические разложения в ЦПТ появились задолго до теоремы Берри-Эссеена, правда, оценки точности этих аппроксимаций, за двумя исключениями, связанными с результатами Я. В. Успенского (их формулировки можно найти в [1], [3, с. 234], [4, с. 95]), долгое время нельзя было доводить до численных значений.

Нас будут интересовать асимптотические разложения в локальных формах ЦПТ. Существуют две локальные формы ЦПТ, одна из них связана с плотностями распределений $P_{n}$ (если они существуют), другая с величинами скачков функций распределения $F_{n}(x)$ в их точках разрыва в случае, когда распределение $P$ решетчато.

Говоря о разложениях для плотностей, мы всегда предполагаем, что для некоторого числа $\nu>0$ выполнено условие

$$
\int_{-\infty}^{\infty}|f(t)|^{\nu} d t<\infty
$$

где $f$ - характеристическая функция распределения $P$. Выполнение этого условия гарантирует при $n \geqslant \nu$ существование непрерывной плотности $p_{n}(x)$ распределения $P_{n}$, которую можно вычислять по формуле обращения

$$
p_{n}(x)=\frac{1}{2 \pi} \int_{-\infty}^{\infty} e^{i t x} f^{n}\left(\frac{t}{\sqrt{n}}\right) d t, \quad-\infty<x<\infty
$$

и справедливость локальной формы ЦПТ: $p_{n}(x) \rightarrow \varphi(x), n \rightarrow \infty$, для всех действительных $x$. Здесь и далее $\varphi(x)=e^{-x^{2} / 2} / \sqrt{2 \pi}-$ плотность стандартного нормального закона.

Из существования плотности $p_{n}$ для некоторого $n$ следует, что $f(t) \rightarrow 0, t \rightarrow \infty$, и число

$$
\alpha(T)=\max \{|f(t)|:|t| \geqslant T\},
$$


которое нам понадобится далее, строго меньше единицы для любого $T>0$.

Говоря о разложениях для величин скачков решетчатых функций распределения, мы всегда предполагаем, что распределение $P$ сосредоточено на некотором множестве, принадлежащем решетке $D=\{a+k h: k=$ $0, \pm 1, \pm 2, \ldots\}$, где $a$ - некоторое действительное число, а $h>0$ - шаг распределения $P$, т.е. максимальное число, которое можно использовать в определении множества $D$. В этом случае для любого натурального $n$ распределение $P_{n}$ сосредоточено на некотором множестве, принадлежащем решетке $D_{n}=\left\{a_{n}+k h / \sqrt{n}: k=0, \pm 1, \pm 2, \ldots\right\}$, где $a_{n}$ - произвольная точка множества $D_{n}$, например, $a_{n}=a \sqrt{n}$. Формулу обращения для решетчатых распределений можно записать в виде

$$
\frac{\sqrt{n}}{h} p_{n}(x)=\frac{1}{2 \pi} \int_{-\pi \sqrt{n} / h}^{\pi \sqrt{n} / h} e^{-i t x} f^{n}\left(\frac{t}{\sqrt{n}}\right) d t, \quad x \in D_{n},
$$

где мы обозначили $p_{n}(x)$ скачок функции распределения $F_{n}(x)$ в точке $x \in D_{n}$. Совпадение этого обозначения с аналогичным обозначением для плотности не должно привести к недоразумениям. В случае решетчатого распределения $P$ число

$$
\alpha(T)=\max \{|f(t)|: T \leqslant|t| \leqslant \pi / h\},
$$

которое нам понадобится далее, также строго меньше единицы для любого $0<T<\pi / h$. Совпадение этого обозначения с аналогичным в случае гладких распределений также не должно вызвать недоразумений.

Правые части формул обращения (2) и (3) отличаются лишь областями интегрирования. Это приводит к тому, что главные части асимптотических разложений для функций $p_{n}(x),-\infty<x<\infty$, в гладком случае, и $\sqrt{n} p_{n}(x) / h, x \in D_{n}$, в случае решетчатых распределений совпадают. Это следует и из результатов К.-Г. Эссеена и Б. В. Гнеденко, см., например, [2], теоремы 13 и 15 параграфа 3 главы VII и комментарии к этой главе. Оценки остаточных частей разложений для гладкого и решетчатого случаев отличаются лишь в одном слагаемом, убывающем при росте $n$ экспоненциально быстро (об этом говорится ниже).

Мы называем главными частями разложений суммы величин, выписанных в явном виде и не содержащих неизвестных функций или постоянных. Остаточными частями разложений мы называем суммы величин, для которых указаны лишь их явные оценки. Если нам потребуется записать сумму главной и остаточной частей разложения, мы в слагаемые остаточной части будем добавлять множители $\gamma$. Этим символом обозначаются различные (даже в пределах одной и той же формулы) функции, абсолютные величины которых не превосходят единицы. Равенство $u(t)=\gamma U(t)$, где $\gamma=\gamma(t)$, эквивалентно неравенству $|u(t)| \leqslant|U(t)|$. 
В этой работе нам понадобятся численные иллюстрации, одно из назначений которых - показать, зачем мы будем модифицировать те или иные разложения. Для этого мы будем использовать симметричное распределение Бернулли (СРБ), сосредоточенное в двух точках -1 и 1 которым приписаны вероятности 0.5. Для СРБ все моменты четных порядков равны единице, оно является решетчатым и его шаг равен двум.

В [5] были получены новые общие результаты об асимптотических разложениях в локальных формах ЦПТ и приведены три следствия этих результатов для распределений $P$ с конечными моментами четвертого, пятого и шестого порядков. Для определенности мы будем говорить о разложениях для плотностей. Для распределений $P$ с конечным моментом шестого порядка главная часть разложения плотности $p_{n}(x), n \geqslant \nu$, содержит, помимо плотности $\varphi(x)$ стандартного нормального закона, произведения $H_{l}(x) \varphi(x)$, где $H_{l}(x), l=3,4, \ldots, 12, l \neq 11$ - многочлены Чебышёва-Эрмита. Коэффициенты при этих функциях зависят от $n$ и от моментов $P$. Всего в главной части используется 11 комбинаций моментных характеристик распределения $P$, из которых 7 связаны с третьим моментом. Оценка остаточной части этого разложения содержит 25 слагаемых, связанных со своими комбинациями моментных характеристик распределения $P$, из которых 16 связаны с третьим моментом и еще 4 с другими моментами $P$ нечетных порядков. Для того, чтобы сделать эти и приводимые ниже разложения сколь-нибудь обозримыми, мы далее рассматриваем только симметричные распределения. В этом случае упомянутое следствие для распределений с конечным моментом шестого порядка имеет следующий вид.

Если для распределения $P$ выполнено условие (1), то в случае $\beta_{6}<\infty$ при $n \geqslant \max (\nu, 5)$ плотность $p_{n}(x)$ при всех действительньх $x$ допускает представление

$$
\begin{aligned}
p_{n}(x)= & \varphi(x)+\frac{\theta_{4}}{4 ! n} H_{4}(x) \varphi(x)+\frac{\theta_{6}^{(4)}}{6 ! n^{2}} H_{6}(x) \varphi(x) \\
& +\frac{n-1}{2 n} \frac{1}{n^{2}}\left(\frac{\theta_{4}}{4 !}\right)^{2} H_{8}(x) \varphi(x)+R+K,
\end{aligned}
$$

гдe

$$
\begin{aligned}
|R| \leqslant & \frac{\beta_{6}}{6 !} \frac{B_{6, n}}{n^{2}}+\frac{\left\|\theta_{8}^{(4)}\right\|}{8 !} \frac{B_{8, n}}{n^{3}} \\
& +\frac{1}{2}\left(\frac{\left|\theta_{4}\right|}{4 !} \frac{\left\|\theta_{6}^{(4)}\right\|}{6 !}+\frac{\beta_{4}+3}{4 !} \frac{\left|\theta_{6}^{(4)}\right|}{6 !}\right) \frac{B_{10, n}}{n^{3}}+\frac{1}{6}\left(\frac{\theta_{4}}{4 !}\right)^{2} \frac{\beta_{4}+3}{4 !} \frac{B_{12, n}}{n^{3}} .
\end{aligned}
$$

О величине $K$ которая при росте $n$ убывает экспоненциально быстро, мы скажем ниже. 
Утверждения, как сформулированные выше, так и те, что приведены ниже, справедливы и для решетчатых распределений. В этом случае в формулировках утверждений нужно снять условие (1), условия $n \geqslant \max (\nu, k)$ следует заменить на $n \geqslant k$, слова «плотность $p_{n}(x) \gg$ нужно заменить на «величина $\sqrt{n} p_{n}(x) / h \gg$, слова «при всех действительных $x \gg$ нужно заменить на «при всех $x \in D_{n} \gg$, в левых частях равенств заменить $p_{n}(x)$ на величину $\sqrt{n} p_{n}(x) / h$ и заменить одно слагаемое из тех, что составляют величину $K$, на другое, о чем пойдет речь ниже. Далее для решетчатых распределений мы будем величину $\sqrt{n} p_{n}(x) / h$ обозначать $\widetilde{p}_{n}(x)$. Из разложений для плотностей довольно просто получить также разложения для функций распределения (см., например, [4, гл. 4, $\S 15])$.

В (4) и далее $H_{l}(x)=(-1)^{l} \varphi^{(l)}(x) / \varphi(x), l=0,1, \ldots$, - многочлены Чебышёва-Эрмита. Первые пять многочленов Чебышёва-Эрмита суть

$$
\begin{gathered}
H_{0}(x) \equiv 1, \quad H_{1}(x)=x, \quad H_{2}(x)=x^{2}-1, \\
H_{3}(x)=x^{3}-3 x, \quad H_{4}(x)=x^{4}-6 x^{2}+3 .
\end{gathered}
$$

Числа

$$
\theta_{l}=\int_{-\infty}^{\infty} H_{l}(x) P(d x),
$$

которые мы называем моментами Чебышёва-Эрмита распределения $P$, можно вычислять по формуле

$$
\frac{\theta_{l}}{l !}=\sum_{j=0}^{[l / 2]} \frac{\alpha_{l-2 j}}{(l-2 j) !} \frac{(-1)^{j}}{2^{j} \cdot j !}, \quad l=0,1, \ldots
$$

Для любого распределения $P$ величина $\theta_{0}=1$. Для стандартного нормального закона все моменты Чебышёва-Эрмита, начиная с первого, равны нулю. Для распределений с нулевым средним и единичной дисперсией $\theta_{1}=\theta_{2}=0, \theta_{3}=\alpha_{3}, \theta_{4}=\alpha_{4}-3$, в частности, для СРБ $\theta_{4}=-2$. Для симметричных распределений могут быть отличны от нуля моменты Чебышёва-Эрмита только четных порядков и предыдущая формула приобретает вид

$$
\frac{\theta_{2 l}}{(2 l) !}=\sum_{j=0}^{l} \frac{\alpha_{2 l-2 j}}{(2 l-2 j) !} \frac{(-1)^{j}}{2^{j} \cdot j !}, \quad l=0,1, \ldots
$$

Числа $\theta_{l}^{(r)}$, которые мы будем называть квазимоментами ЧебышёваЭрмита, вычисляются по той же формуле, что и $\theta_{l}$, но в сумме, указанной выше, слагаемые, связанные со степенными моментами порядков больших $r$, опускаются. В частности, для СРБ $\theta_{6}^{(4)} / 6 !=\left(-\alpha_{4}+2\right) / 48=1 / 48$ и $\theta_{8}^{(4)} / 8 !=-1 / 384$. Числа $\left\|\theta_{l}\right\|$ и $\left\|\theta_{l}^{(r)}\right\|$ при четных $l$ вычисляются по 
тем же формулам, что $\theta_{l}$ и $\theta_{l}^{(r)}$, в которых $(-1)^{j}$ заменяется на 1 . В частности, для СРБ $\left\|\theta_{6}^{(4)}\right\| / 6 !=5 / 48$ и $\left\|\theta_{8}^{(4)}\right\| / 8 !=7 / 384$.

Разумеется, всюду вместо $\beta_{2 l}, l$ - натуральное, можно писать $\alpha_{2 l}$, но нам удобно использовать для старшего из моментов обозначение абсолютного момента.

Числа $B_{l, n}$ определяются равенствами

$$
B_{l, n}=\frac{1}{2 \pi} \int_{-T \sqrt{n}}^{T \sqrt{n}}|t|^{l} \mu^{n-1}\left(\frac{t}{\sqrt{n}}\right) d t, \quad l=4,5, \ldots,
$$

где $\mu(t)=\max \left\{|f(t)|, e^{-t^{2} / 2}\right\}$. На параметр $T$, выбор которого находится в нашем распоряжении, в случае гладких распределений никаких формальных ограничений не налагается, однако для конкретных распределений ограничения могут появиться, если мы захотим получить хорошие оценки для $B_{l, n}$. В случае решетчатых распределений в качестве $T$ можно брать любое положительное число, не превосходящее $\pi / h$. В обоих случаях для распределений с конечным четвертым моментом существует значение $T$, при котором $B_{l, n} \rightarrow B_{l}, n \rightarrow \infty$, где $B_{l}-$ абсолютный момент $l$-го порядка стандартного нормального закона, деленный на $\sqrt{2 \pi}$ (подробнее см. в [5]). Далее мы считаем, что параметр $T$ выбран именно таким образом.

В разложениях, как в приведенном выше, так и в тех, что будут получены далее, величина $K$ является суммой слагаемых, первое из которых не превосходит

$$
\frac{\sqrt{n}}{\pi} \alpha^{n-\nu}(T) \int_{T}^{\infty}|f(t)|^{\nu} d t
$$

в случае гладких распределений и

$$
\frac{\sqrt{n}}{\pi} \alpha^{n}(T)\left(\frac{\pi}{h}-T\right)
$$

в случае решетчатых распределений. Определения чисел $\alpha(T)$ в этих случаях несколько отличаются друг от друга, но в обоих случаях это слагаемое при росте $n$ стремится к нулю экспоненциально быстро. Второе слагаемое в $K$ не превосходит величины

$$
\frac{1}{\pi T \sqrt{n}} e^{-T^{2} n / 2}
$$

которая также при росте $n$ стремится к нулю экспоненциально быстро. Оценки остальных слагаемых из $K$ получаются из главных частей разложений заменой множителей перед произведениями $H_{l}(x) \varphi(x), l \geqslant 4$, их абсолютными значениями, а самих произведений — величинами

$$
\frac{1}{\pi} \int_{T \sqrt{n}}^{\infty} t^{l} e^{-t^{2} / 2} d t
$$


которые при росте $n$ также стремятся к нулю экспоненциально быстро.

В оценках остаточных частей разложений, которые рассматриваются в этой работе, ровно одно слагаемое, первое, имеет правильный порядок убывания при росте $n$. Будем называть это слагаемое «основным», а остальные слагаемые, они при росте $n$ стремятся к нулю быстрее «основного», будем называть «вспомогательными». Причина, по которой мы здесь используем кавычки, станет ясна из дальнейшего. «Основные» слагаемые в оценках остаточных частей разложений, как приведенного выше, так и тех, что будут получены далее в этой работе, неулучшаемы в том смысле, что если их умножить на произвольную постоянную, которая меньше единицы (и сколь угодно близка к ней), то оценка станет неверной, начиная с некоторого $n$. Об этом говорится в $[5]$.

Оценки остаточных частей разложений, которые будут получены далее, довольно громоздки, они являются суммами многих слагаемых. Для того чтобы получить представление о вкладах этих слагаемых в сумму, проще всего вычислить их для какого-либо конкретного распределения. В качестве такого распределения мы возьмем СРБ, характеристическая функция которого $f(t)=\cos t$. На интервале $(-\pi / 2, \pi / 2)$ справедливо неравенство $0<\cos t \leqslant e^{-t^{2} / 2}$. Для СРБ в качестве величины $T$ можно взять число $\pi / 2$. При таком выборе $T$ слагаемое в оценке $K$, связанное с $\alpha(T)$, станет равным нулю, а в оценках слагаемых, составляющих величину $K$, появятся множители $e^{-\pi^{2} n / 8}$, что сделает их пренебрежимо малыми по сравнению со слагаемыми, входящими в оценки величин $R$. На интервале $(-\pi / 2, \pi / 2)$ функция $\mu(t)=e^{-t^{2} / 2}$, поэтому

$$
B_{l, n}=\frac{1}{2 \pi} \int_{-\pi \sqrt{n} / 2}^{\pi \sqrt{n} / 2}|t|^{l} \exp \left(-(n-1) \frac{t^{2}}{2 n}\right) d t \leqslant\left(\frac{n}{n-1}\right)^{(l+1) / 2} B_{l} .
$$

Мы получим полезную информации о точности аппроксимации, которую гарантируют асимптотические разложения, если вычислим лишь приближенные значения оценок их остаточных частей. Такие оценки мы будем получать, заменяя величины $B_{l, n}$ их асимптотическими значениями $B_{l}$. Так полученные оценки величин $R$ можно назвать прикидочными. Поскольку мы, как правило, будем вычислять только прикидочные оценки, слово «прикидочные» будет обычно опускаться, это не должно привести к недоразумениям. Величинами $K$ для СРБ мы будем пренебрегать.

Главная часть разложения (4) для $\widetilde{p}_{n}(x), x \in D_{n}$, для СРБ есть

$$
\varphi(x)\left(1-\frac{1}{12 n} H_{4}(x)+\frac{1}{48 n^{2}} H_{6}(x)+\frac{n-1}{2 n} \frac{1}{144 n^{2}} H_{8}(x)\right) .
$$

Вычисляя оценку остаточной части этого разложения, мы получим ве- 
личину, которую, несколько загрубляя, можно записать в виде

$$
\frac{\beta_{6}}{120 n^{2}}+\frac{0.77}{n^{3}}+\frac{2.3}{n^{3}}+\frac{0.81}{n^{3}}=\frac{\beta_{6}}{120 n^{2}}+\frac{3.88}{n^{3}} \approx \frac{1}{120 n^{2}}\left(1+\frac{466}{n}\right) .
$$

Мы видим, что при $n$ порядка нескольких десятков и даже немногих сотен в оценке остаточной части указанного разложения «вспомогательные» слагаемые играют главную роль. Это нас и не устраивает.

Далее эта оценка будет уточняться, будут получены другие разложения, в оценках остаточных частей которых соотношения между «основным» и суммой «вспомогательных» слагаемых лучше, чем в указанной. Отметим, однако, что при $n=1600$, т.е. когда оценка теоремы Берри-Эссеена еще не может гарантировать ни для какого распределения точность аппроксимации, лучшую $10^{-2}$, для СРБ правая часть (5), только что вычисленная, не превосходит $4.3 \cdot 10^{-9}$. Столь большие значения $n$ для нас не очень интересны, нас интересуют $n$ порядка нескольких десятков. Нас не смущает сравнение точности аппроксимаций в локальной и интегральной формах ЦПТ, поскольку если для данного распределения справедлива ЦПТ в локальной форме, то в ней оценки точности аппроксимации оказываются несколько хуже, чем можно получить в интегральной форме ЦПТ.

Стоит отметить, что при построении оценки (5) рассуждения в некоторых местах были достаточно грубыми, поэтому грубой оказалась и оценка. Так, при оценке разности характеристических функций $f(t / \sqrt{n})$ и $g(t / \sqrt{n})(g$ - характеристическая функция стандартного нормального закона) использовалось почти очевидное неравенство

$$
\left|f\left(\frac{t}{\sqrt{n}}\right)-g\left(\frac{t}{\sqrt{n}}\right)\right| \leqslant \frac{\beta_{4}+3}{4 !}\left(\frac{t}{\sqrt{n}}\right)^{4}
$$

для распределений с конечным четвертым и нулевым третьим моментом, в то время как для распределений с конечным шестым моментом и нулевыми моментами третьего и пятого порядков справедливо более точное неравенство

$$
\left|f\left(\frac{t}{\sqrt{n}}\right)-g\left(\frac{t}{\sqrt{n}}\right)\right| \leqslant \frac{\theta_{4}}{4 !}\left(\frac{t}{\sqrt{n}}\right)^{4}+\left(\frac{\beta_{6}}{6 !}+\frac{1}{48}\right)\left(\frac{1}{\sqrt{n}}\right)^{6}
$$

(об этом говорится в доказательстве леммы 2). Использование последнего неравенства позволяет для СРБ в оценках остаточных частей в два раза уменьшить значения слагаемых, содержащих множитель $\beta_{4}+3$, правда, при этом в оценках появятся дополнительные слагаемые, убывающие при росте $n$ быстрее остальных. Еще одна причина грубости оценок связана с тем, что в суммах, составляющих величины $\left\|\theta_{l}^{(r)}\right\|$, теряется чередование знаков слагаемых, которое есть в суммах, дающих 
величины $\theta_{l}^{(r)}$. Это чередование знаков можно отчасти восстановить, заменив в оценках $\left\|\theta_{l}^{(r)}\right\|$ на сумму $\left|\theta_{l}^{(r)}\right|$ и слагаемого, связанного с $\left\|\theta_{l+2}^{(r)}\right\|$. Под знаком модуля чередование знаков сохраняется и величина $\left|\theta_{l}^{(r)}\right|$ может оказаться существенно меньше $\left\|\theta_{l}^{(r)}\right\|$ (и мы это только что видели, для СРБ величина $\left\|\theta_{6}^{(4)}\right\|$ в пять раз больше $\left.\left|\theta_{6}^{(4)}\right|\right)$. В оценке, правда, появится величина, связанная с $\left\|\theta_{l+2}^{(r)}\right\|$, но она будет убывать при росте $n$ быстрее той, что связана с $\left|\theta_{l}^{(r)}\right|$.

Учет сказанного выше приводит к следующему утверждению.

Теорема 1. Для любого симметричного распределения $P$ с нулевьм средним и единичной дисперсией, такого, что для некоторого $\nu>0$ выполнено условие (1), при конечности момента $\beta_{6}$ для всех действительньх $x$ и всех $n \geqslant \max (\nu, 3)$ плотность $p_{n}(x)$ допускает представление (4), в котором для величинь $R$ справедлива оченка

$$
\begin{aligned}
|R| \leqslant & \frac{\beta_{6}}{6 !} \frac{B_{6, n}}{n^{2}}+\frac{1}{2} \frac{\left|\theta_{4}\right|}{4 !} \frac{\beta_{6}}{6 !} \frac{B_{10, n}}{n^{3}}+\frac{1}{2} \frac{\left|\theta_{6}^{(4)}\right|}{6 !}\left(\frac{\beta_{6}}{6 !}+\frac{1}{48}\right) \frac{B_{12, n}}{n^{4}} \\
& +\frac{1}{6} \frac{\left|\theta_{4}\right|}{4 !}\left(\frac{\beta_{6}}{6 !}+\frac{1}{48}\right) \frac{B_{14, n}}{n^{4}}+\frac{\left|\theta_{8}^{(4)}\right|}{8 !} \frac{B_{8, n}}{n^{3}}+\frac{\left|\theta_{4}\right|}{4 !} \frac{\left|\theta_{6}^{(4)}\right|}{6 !} \frac{B_{10, n}}{n^{3}} \\
& +\frac{1}{6}\left|\frac{\theta_{4}}{4 !}\right|^{3} \frac{B_{12, n}}{n^{3}}+\frac{\left\|\theta_{10}^{(4)}\right\|}{10 !} \frac{B_{10, n}}{n^{4}}+\frac{1}{2} \frac{\left|\theta_{4}\right|}{4 !} \frac{\left\|\theta_{8}^{(4)}\right\|}{8 !} \frac{B_{12, n}}{n^{4}} .
\end{aligned}
$$

Это же представление справедливо для функиий $\widetilde{p}_{n}(x), x \in D_{n}$, в случае решетчатых распределений. B cлучае решетчатых распределений условие (1) снимается и приведенное выше ограничение на $n$ следует заменить на $n \geqslant 3$.

Доказательство этого утверждения приведено ниже. В оценке (6) сначала выписаны слагаемые, связанные с $\beta_{6}$, затем - слагаемые, убывающие при росте $n$ как $n^{-3}$ и не связанные с $\beta_{6}$ (эту группу слагаемых мы будем называть основной), а затем - остальные слагаемые.

Мы уже приводили для СРБ значения всех величин из правой части последнего неравенства, за исключением $\left\|\theta_{10}^{(4)}\right\|$. Легко проверить, что для СРБ $\left\|\theta_{10}^{(4)}\right\| / 10 !=7 / 2880$.

Вычисление для СРБ части оценки (6), связанной с $\beta_{6}$, приводит к величине, не превосходящей

$$
\frac{\beta_{6}}{120 n^{2}}+\frac{0.022}{n^{3}}+\frac{0.97}{n^{4}}+\frac{1.4}{n^{4}}=\frac{1}{120 n^{2}}\left(1+\frac{2.64}{n}+\frac{285}{n^{2}}\right) .
$$

Вычисление части оценки правой части (6), связанной с основной группой слагаемых, приводят к величине, приближенно равной

$$
\frac{0.11}{n^{3}}+\frac{0.66}{n^{3}}+\frac{0.4}{n^{3}}=\frac{1.17}{n^{3}}=\frac{1}{120 n^{2}} \cdot \frac{140.4}{n} .
$$


Вычисление части оценки правой части (6), связанной с оставшимися слагаемыми, приводит к величине, не превосходящей

$$
\frac{0.92}{n^{4}}+\frac{3.16}{n^{4}}=\frac{4.08}{n^{4}}=\frac{1}{120 n^{2}} \cdot \frac{490}{n^{2}} .
$$

Мы видим, что для СРБ при $n$ порядка 100 основная группа слагаемых вносит довольно существенный вклад в оценку. Единственный способ убрать ее из оценки остаточной части разложения - «перевести» в главную часть, при этом, правда, в оценке остаточной части появятся дополнительные слагаемые, убывающие при росте $n$ быстрее, чем $n^{-3}$. Справедливо следующее утверждение.

Теорема 2. Для любого симметричного распределения $P$ с нулевьм средним и единичной дисперсией, такого, что для некоторого $\nu>0$ выполнено условие (1), при конечности момента $\beta_{6}$ для всех действительньх $x$ и всех $n \geqslant \max (\nu, 4)$ плотность $p_{n}(x)$ допускает представление

$$
\begin{aligned}
p_{n}(x)= & \varphi(x)+\frac{\theta_{4}}{4 ! n} H_{4}(x) \varphi(x)+\frac{\theta_{6}^{(4)}}{6 ! n^{2}} H_{6}(x) \varphi(x) \\
& +\frac{n-1}{2 n} \frac{1}{n^{2}}\left(\frac{\theta_{4}}{4 !}\right)^{2} H_{8}(x) \varphi(x)+\frac{\theta_{8}^{(4)}}{8 ! n^{3}} H_{8}(x) \varphi(x) \\
& +\frac{n-1}{n} \frac{1}{n^{3}} \frac{\theta_{4}}{4 !} \frac{\theta_{6}^{(4)}}{6 !} H_{10}(x) \varphi(x) \\
& +\frac{(n-1)(n-2)}{6 n^{2}} \frac{1}{n^{3}}\left(\frac{\theta_{4}}{4 !}\right)^{3} H_{12}(x) \varphi(x)+R+K,
\end{aligned}
$$

$2 \partial e$

$$
\begin{aligned}
|R| \leqslant & \frac{\beta_{6}}{6 !} \frac{B_{6, n}}{n^{2}}+\frac{1}{2} \frac{\left|\theta_{4}\right|}{4 !} \frac{\beta_{6}}{6 !} \frac{B_{10, n}}{n^{3}}+\frac{1}{2} \frac{\left|\theta_{6}^{(4)}\right|}{6 !} \frac{\beta_{6}}{6 !} \frac{B_{12, n}}{n^{4}}+\frac{1}{6}\left(\frac{\theta_{4}}{4 !}\right)^{2} \frac{\beta_{6}}{6 !} \frac{B_{14, n}}{n^{4}} \\
& +\frac{1}{3} \frac{\left|\theta_{4}\right|}{4 !} \frac{\left|\theta_{6}^{(4)}\right|}{6 !}\left(\frac{\beta_{6}}{6 !}+\frac{1}{48}\right) \frac{B_{16, n}}{n^{5}}+\frac{1}{2}\left(\frac{\beta_{6}}{6 !}+\frac{1}{48}\right) \frac{\left|\theta_{8}^{(4)}\right|}{8 !} \frac{B_{14, n}}{n^{5}} \\
& +\frac{1}{24}\left|\frac{\theta_{4}}{4 !}\right|^{3}\left(\frac{\beta_{6}}{6 !}+\frac{1}{48}\right) \frac{B_{18, n}}{n^{5}}+\frac{\left|\theta_{10}^{(4)}\right|}{10 !} \frac{B_{10, n}}{n^{4}}+\frac{\left|\theta_{4}\right|}{4 !} \frac{\left|\theta_{8}^{(4)}\right|}{8 !} \frac{B_{12, n}}{n^{4}} \\
& +\frac{1}{2}\left(\frac{\theta_{6}^{(4)}}{6 !}\right)^{2} \frac{B_{12, n}}{n^{4}}+\frac{1}{2}\left(\frac{\theta_{4}}{4 !}\right)^{2} \frac{\left|\theta_{6}^{(4)}\right|}{6 !} \frac{B_{14, n}}{n^{4}}+\frac{1}{24}\left(\frac{\theta_{4}}{4 !}\right)^{4} \frac{B_{16, n}}{n^{4}} \\
& +\frac{\left\|\theta_{12}^{(4)}\right\|}{12 !} \frac{B_{12, n}}{n^{5}}+\frac{1}{2} \frac{\left|\theta_{4}\right|}{4 !} \frac{\left\|\theta_{10}^{(4)}\right\|}{10 !} \frac{B_{14, n}}{n^{5}}+\frac{1}{2} \frac{\left|\theta_{6}^{(4)}\right|}{6 !} \frac{\left\|\theta_{8}^{(4)}\right\|}{8 !} \frac{B_{14, n}}{n^{5}} \\
& +\frac{1}{6}\left(\frac{\theta_{4}}{4 !}\right)^{2} \frac{\left\|\theta_{8}^{(4)}\right\|}{8 !} \frac{B_{16, n}}{n^{5}},
\end{aligned}
$$

а величина $K$ является суммой слагаемьх, убывающих экспоненциально быстро при росте $n$, вид этих слагаемьх приведен в доказательстве теоремь. 
Это же представление справедливо для функиий $\widetilde{p}_{n}(x), x \in D_{n}$, в случае решетчатых распределении. B случае решетчатых распределений условие (1) снимается и приведенное выше ограничение на $n$ следует заменить на $n \geqslant 4$.

Kaк и в правой части формулы (6), в правой части (8) сначала выписаны слагаемые, связанные с $\beta_{6}$. Затем идут слагаемые, убывающие при росте $n$ как $n^{-4}$, и не связанные с $\beta_{6}$, мы вновь назовем эту группу слагаемых основной, и затем - остальные слагаемые. В (8) появилась величина $\left\|\theta_{12}^{(4)}\right\| / 12$ !, которая нам еще не встречалась, нетрудно проверить, что для СРБ она равна $1 / 3840$.

Вычислим для СРБ часть оценки (8) связанную с величиной $\beta_{6}$. Эта часть не превосходит

$$
\frac{\beta_{6}}{120 n^{2}}+\frac{0.022}{n^{3}}+\frac{0.147}{n^{4}}+\frac{19.39}{n^{5}} \approx \frac{1}{120 n^{2}}\left(1+\frac{2.64}{n}+\frac{17.64}{n^{2}}+\frac{2327}{n^{3}}\right) .
$$

Сумма слагаемых основной группы приближенно равна

$$
\frac{1}{n^{4}}(0.066+0.903+0.903+3.91+1.63) \approx \frac{7.5}{n^{4}}=\frac{1}{120 n^{2}} \cdot \frac{900}{n^{2}} .
$$

Сумма оставшихся слагаемых не превосходит

$$
\frac{1}{n^{5}}(1.1+5.48+10.27+17.1)<\frac{34}{n^{5}}=\frac{1}{120 n^{2}} \cdot \frac{4080}{n^{3}} .
$$

Легко видеть, что для СРБ при $n=30$ вклад в оценку основной группы слагаемых совпадает с вкладом «основного» слагаемого, суммарный вклад остальных слагаемых существенно меньше.

Основную группу слагаемых из правой части (8) также можно перевести в главную часть разложения, но это приводит к очень громоздким формулам: главная часть разложения будет состоять из 12 слагаемых, а оценка величины $R$ - из 21 слагаемого.

Заканчивая обсуждение разложений для СРБ, скажем, что в точке $x=0$ разность между $\widetilde{p}_{n}(x)$ и главной частью разложения (4) при $n=36$ составляет $-2.96 \ldots \cdot 10^{-6}$.

Приступим к доказательству приведенных выше утверждений.

Д о к а з а т е л ь с т в а теорем основаны на использовании формул обращения для преобразований Фурье и на представлении функции $f^{n}(t / \sqrt{n})$ в формах, указанных в леммах 2 и 3 , формулировки и доказательства которых приведены ниже. Доказательства теорем дают алгоритм, позволяющий из разложений $f^{n}(t / \sqrt{n})$ получать разложения для $p_{n}(x)$ или $\widetilde{p}_{n}(x)$ вместе с оценками остаточных частей. Для определенности мы будем рассматривать случай гладких распределений. Для решетчатых распределений мы укажем лишь то место, где появляется 
отличие в оценке остаточной части разложения по сравнению с гладким случаем.

Формула обращения (2), которая справедлива и для стандартного нормального закона, позволяет при $n \geqslant \nu$ записать разность $p_{n}(x)-\varphi(x)$ в виде

$$
p_{n}(x)-\varphi(x)=\frac{1}{2 \pi} \int_{-\infty}^{\infty} e^{-i t x}\left(f^{n}\left(\frac{t}{\sqrt{n}}\right)-e^{-t^{2} / 2}\right) d t, \quad-\infty<x<\infty .
$$

За редкими исключениями, мы можем получать хорошие оценки функции $f(t)$, а вместе с ней и функции $\mu(t)=\max \left(|f(t)|, e^{-t^{2} / 2}\right)$, лишь на некотором конечном интервале $(-T, T), T>0$. В связи с этим нам приходится разбивать область интегрирования в правой части (9) на интервал $(-T \sqrt{n}, T \sqrt{n})$ и его дополнение. Абсолютная величина интеграла по этому дополнению не превосходит

$$
\frac{1}{\pi} \int_{T \sqrt{n}}^{\infty}\left|f\left(\frac{t}{\sqrt{n}}\right)\right|^{n} d t+\frac{1}{\pi} \int_{T \sqrt{n}}^{\infty} e^{-t^{2} / 2} d t .
$$

Первая из этих величин равна

$$
\frac{\sqrt{n}}{\pi} \int_{T}^{\infty}|f(t)|^{n-\nu}|f(t)|^{\nu} d t \leqslant \frac{\sqrt{n}}{\pi} \alpha^{n-\nu}(T) \int_{T}^{\infty}|f(t)|^{\nu} d t
$$

что при росте $n$ стремится к нулю экспоненциально быстро, поскольку $\alpha(T)$ строго меньше единицы для любого $T>0$. Вторая из этих величин, как хорошо известно, не превосходит

$$
\frac{1}{\pi T \sqrt{n}} e^{-T^{2} n / 2}
$$

и нам осталось лишь разобраться с интегралом

$$
\frac{1}{2 \pi} \int_{-T \sqrt{n}}^{T \sqrt{n}} e^{-i t x}\left(f^{n}\left(\frac{t}{\sqrt{n}}\right)-e^{-t^{2} / 2}\right) d t
$$

Для решетчатых распределений формула обращения (3) позволяет утверждать, что

$$
\frac{\sqrt{n}}{h} p_{n}(x)-\varphi(x)=\frac{1}{2 \pi} \int_{-\pi \sqrt{n} / h}^{\pi \sqrt{n} / h} e^{-i t x} f^{n}\left(\frac{t}{\sqrt{n}}\right) d t-\frac{1}{2 \pi} \int_{-\infty}^{\infty} e^{-i t x} e^{-t^{2} / 2} d t .
$$

И в этом случае мы, за редкими исключениями, можем получать хорошие оценки функции $f(t)$, а вместе с ней и функции $\mu(t)$, лишь на некотором интервале $(-T, T), 0<T<\pi / h$. Поэтому и здесь нам придется разбивать область интегрирования в первом интеграле в правой части на интервал $(-T \sqrt{n}, T \sqrt{n})$ и его дополнение до $(-\pi \sqrt{n} / h, \pi \sqrt{n} / h)$. 
В связи с этим область интегрирования во втором интеграле в правой части также разбивается на интервал $(-T \sqrt{n}, T \sqrt{n})$ и его дополнение до всей действительной прямой. В соответствии с этим, правая часть последнего равенства равна сумме величины (10) и величины, модуль которой не превосходит

$$
\frac{1}{\pi} \int_{T \sqrt{n}}^{\pi \sqrt{n} / h}\left|f\left(\frac{t}{\sqrt{n}}\right)\right|^{n} d t+\frac{1}{\pi} \int_{T \sqrt{n}}^{\infty} e^{-t^{2} / 2} d t
$$

Второе слагаемое - то же самое, что и в предыдущем случае, а первое равно

$$
\frac{\sqrt{n}}{\pi} \int_{T}^{\pi / h}|f(t)|^{n} d t \leqslant \frac{\sqrt{n}}{\pi} \alpha^{n}(T)\left(\frac{\pi}{h}-T\right),
$$

где $\alpha(T)<1$, что вновь при росте $n$ стремится к нулю экспоненциально быстро. Это - единственное место, где возникает различие в оценках остаточных частей разложений для гладкого и решетчатого случаев.

Рассмотрим величину (10). Ее вид один и тот же как для гладких, так и для решетчатых распределений. Леммы 2 и 3 , приведенные ниже, позволяют представить разность $f^{n}(t / \sqrt{n})-e^{-t^{2} / 2}$ в виде суммы величин, которые либо имеют вид $a(n, P) e^{-t^{2} / 2}(i t)^{l}$, либо имеют вид $\gamma b(n, P) \mu^{n-1}(t / \sqrt{n}) t^{l}$, где $l$ - натуральное число и $\gamma=\gamma(t)$ - функции, модули которых не превосходят единицы.

Сумма величин вида $a(n, P) e^{-t^{2} / 2}(i t)^{l}$ составляет главную часть разложения разности $f^{n}(t / \sqrt{n})-e^{-t^{2} / 2}$. Очевидно, что для каждой из этих величин

$$
\frac{1}{2 \pi} \int_{-T \sqrt{n}}^{T \sqrt{n}} e^{-i t x} a(n, P) e^{-t^{2} / 2}(i t)^{l} d t=a(n, P) \frac{1}{2 \pi} \int_{-T \sqrt{n}}^{T \sqrt{n}} e^{-i t x} e^{-t^{2} / 2}(i t)^{l} d t .
$$

Правая часть этого равенства в силу хорошо известных соотношений

$$
\frac{1}{2 \pi} \int_{-\infty}^{\infty} e^{-i t x} e^{-t^{2} / 2}(i t)^{l} d t=H_{l}(x) \varphi(x), \quad-\infty<x<\infty,
$$

есть

$$
a(n, P) H_{l}(x) \varphi(x)+K
$$

где

$$
|K| \leqslant|a(n, P)| \frac{1}{\pi} \int_{T \sqrt{n}}^{\infty} t^{l} e^{-t^{2} / 2} d t,
$$

что при росте $n$ стремится к нулю экспоненциально быстро. Сумму первых слагаемых из (11) по всем слагаемым главной части разложения разности $f^{n}(t / \sqrt{n})-e^{-t^{2} / 2}$ мы включим в главную часть разложения $p_{n}(x)$. Сумма величин $K$ из (11) по всем слагаемым главной части разложения разности $f^{n}(t / \sqrt{n})-e^{-t^{2} / 2}$ войдет в остаточную часть разложения $p_{n}(x)$. 
Сумма величин $\gamma b(n, P) \mu^{n-1}(t / \sqrt{n}) t^{l}$ составляет остаточную часть разложения разности $f^{n}(t / \sqrt{n})-e^{-t^{2} / 2}$. Интегралы от этих величин войдут в остаточную часть разложения $p_{n}(x)$. Каждый из таких интегралов внесет в остаточную часть разложения $p_{n}(x)$ вклад, не превосходящий

$$
|b(n, P)| \frac{1}{2 \pi} \int_{-T \sqrt{n}}^{T \sqrt{n}} \mu^{n-1}(t / \sqrt{n})|t|^{l} d t=|b(n, P)| B_{l, n} .
$$

На этом мы закончим доказательство теорем. Сказанное выше позволяет, глядя на разложение функции $f^{n}(t / \sqrt{n})$, сразу выписать как главную часть разложения $p_{n}(x),-\infty<x<\infty$, для гладких распределений или $\widetilde{p}_{n}(x), x \in D_{n}$, для решетчатых распределений, так и оценку остаточной части. Для завершения доказательства теоремы 1 нужно воспользоваться леммой 2 , а для завершения доказательства теоремы 2 - леммой 3 .

Лемма 1. Для симметричных распределений с единичной дисперсией и конечньм моментом порядка $2 k+2, k$ - натуральное, функиия $e^{t^{2} / 2} f(t)$ допускает следуюшие представления

$$
\begin{aligned}
e^{t^{2} / 2} f(t)= & 1+\sum_{l=2}^{k} \frac{\theta_{2 l}}{(2 l) !}(i t)^{2 l}+\sum_{l=k+1}^{k+s} \frac{\theta_{2 l}^{(2 k)}}{(2 l) !}(i t)^{2 l} \\
& +\gamma e^{t^{2} / 2} \frac{\beta_{2 k+2}}{(2 k+2) !} t^{2 k+2}+\gamma e^{t^{2} / 2} \frac{\left\|\theta_{2(k+s+1)}^{(2 k)}\right\|}{(2 k+2 s+2) !} t^{2 k+2 s+2},
\end{aligned}
$$

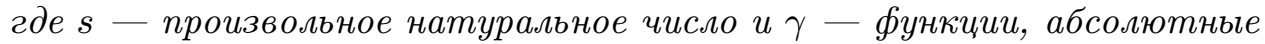
величинь которых не превосходят единицьл.

3 а м е ч а н и е. Утверждение леммы справедливо и при $s=0$. В этом случае в правой части последнего равенства следует опустить вторую сумму по $l$, а сумму двух последних слагаемых можно записать каK

$$
\gamma e^{t^{2} / 2} \frac{\left\|\theta_{2 k+2}\right\|}{(2 k+2) !} t^{2 k+2}
$$

Д о к а з а т е л ь с в о. Доказательство леммы основано на хорошо известном разложении характеристической функции $f(t)$ в отрезок ряда Тейлора, которое мы запишем в виде

$$
f(t)=\sum_{l=0}^{k} \frac{\alpha_{2 l}}{(2 l) !}(i t)^{2 l}+\gamma \frac{\beta_{2 k+2}}{(2 k+2) !} t^{2 k+2},
$$

где $\gamma$ - функция, абсолютная величина которой не превосходит единицы. Произведение

$$
e^{t^{2} / 2} f(t)=e^{t^{2} / 2} \sum_{l=0}^{k} \frac{\alpha_{2 l}}{(2 l) !}(i t)^{2 l}+\gamma e^{t^{2} / 2} \frac{\beta_{2 k+2}}{(2 k+2) !} t^{2 k+2}
$$




$$
\begin{aligned}
& =e^{-(i t)^{2} / 2} \sum_{l=0}^{k} \frac{\alpha_{2 l}}{(2 l) !}(i t)^{2 l}+\gamma e^{t^{2} / 2} \frac{\beta_{2 k+2}}{(2 k+2) !} t^{2 k+2} \\
& =\sum_{j=0}^{\infty} \frac{(-1)^{j}(i t)^{2 j}}{2^{j} j !} \sum_{l=0}^{k} \frac{\alpha_{2 l}}{(2 l) !}(i t)^{2 l}+\gamma e^{t^{2} / 2} \frac{\beta_{2 k+2}}{(2 k+2) !} t^{2 k+2} .
\end{aligned}
$$

Произведение ряда для экспоненты и главной части разложения $f(t)$ в отрезок ряда Тейлора можно вычислять с помощью таблицы, состоящей из $k+1$ столбца и бесконечно многих строк, в заголовках столбцов которой стоят слагаемые из главной части разложения $f(t)$, в заголовках строк - слагаемые из ряда для экспоненты, а элементом, стоящим в $l$-м столбце и $j$-й строке является произведение их заголовков, т.е. величина

$$
\frac{\alpha_{2 l}}{(2 l) !} \frac{(-1)^{j}}{2^{j} j !}(i t)^{2 l+2 j}, \quad l=0,1, \ldots, k, \quad j=0,1, \ldots
$$

Ясно, что сумма всех элементов таблицы совпадает с упомянутым выше произведением. Очевидно, что левый верхний элемент таблицы — число 1. Скажем, что это - элемент тривиальной диагонали. Диагонали строятся справа - налево, сверху — вниз. Легко понять, что сумма элементов $l$-й нетривиальной диагонали, т.е. сумма (по $j$ ) элементов, стоящих в $(l-j)$-м столбце и $j$-й строке, $l=1, \ldots, k, j=0,1, \ldots, l$, есть

$$
\sum_{j=0}^{l} \frac{\alpha_{2 l-2 j}}{(2 l-2 j) !} \frac{(-1)^{j}}{2^{j} j !}(i t)^{2 l}=\frac{\theta_{2 l}}{(2 l) !}(i t)^{2 l} .
$$

Отметим, что сумма элементов первой нетривиальной диагонали равна нулю, так как мы рассматриваем распределения с единичным вторым моментом.

Все эти величины мы включим в главную часть разложения произведения $e^{t^{2} / 2} f(t)$. Мы можем включить в главную часть разложения этого произведения любое число $s$ неполных диагоналей таблицы. Нетрудно проверить, что сумма элементов $l$-й неполной диагонали совпадает с $\theta_{2 l}^{(2 k)}(i t)^{2 l} /(2 l) !, k+1 \leqslant l \leqslant k+s$.

Оценим абсолютную величину суммы элементов таблицы, оставшихся после того как мы включили в главную часть разложения функции $e^{t^{2} / 2} f(t)$ суммы элементов тривиальной диагонали, $k$ нетривиальных полных диагоналей и $s$ неполных диагоналей. Легко видеть, что сумма элементов, оставшихся в $l$-м столбце, $l=0,1, \ldots, k$, есть

$$
\begin{aligned}
& \sum_{j=k+s+1-l}^{\infty} \frac{\alpha_{2 l}}{(2 l) !}(i t)^{2 l} \frac{(-1)^{j}}{2^{j} j !}(i t)^{2 j} \\
& \quad=(-1)^{k+s-l+1} \frac{\alpha_{2 l}}{(2 l) !}(i t)^{2 k+2 s+2} \sum_{j=0}^{\infty} \frac{(-1)^{j}(i t)^{2 j}}{2^{k+s-l+1+j}(k+s-l+1+j) !} .
\end{aligned}
$$


Абсолютная величина правой части этого равенства не превосходит

$$
\begin{aligned}
t^{2 k+2 s+2} & \frac{\alpha_{2 l}}{(2 l) !} \frac{1}{2^{k+s-l+1}(k+s-l+1) !} \sum_{j=0}^{\infty} \frac{t^{2 j}}{2^{j} j !} \\
=e^{t^{2} / 2} & \frac{\alpha_{2 l}}{(2 l) !} \frac{1}{2^{k+s-l+1}(k+s-l+1) !} t^{2 k+2 s+2} .
\end{aligned}
$$

Просуммируем величины

$$
\frac{\alpha_{2 l}}{(2 l) !} \frac{1}{2^{k+s-l+1}(k+s-l+1) !}
$$

по $0 \leqslant l \leqslant k$, перейдя к новой переменной суммирования $j=k+s-l+1$, $s+1 \leqslant j \leqslant k+s+1$. Мы получим

$$
\sum_{j=s+1}^{k+s+1} \frac{\alpha_{2 k+2 s+2-2 j}}{(2 k+2 s+2-2 j) !} \frac{1}{2^{j} j !}=\frac{\left\|\theta_{2 k+2 s+2}^{(2 k)}\right\|}{(2 k+2 s+2) !} .
$$

На этом мы завершим доказательство леммы.

Лемма 2. Для функиии $f^{n}(t / \sqrt{n})$, где $f$ - характеристическая функиия симметричного распределения с единичной дисперсией и конечным моментом шестого порядка при любом натуральном $n \geqslant 3$ справедливо следующее представление:

$$
\begin{aligned}
& f^{n}\left(\frac{t}{\sqrt{n}}\right)=e^{-t^{2} / 2}+\frac{\theta_{4}}{4 ! n}(i t)^{4} e^{-t^{2} / 2}+\frac{\theta_{6}^{(4)}}{6 ! n^{2}}(i t)^{6} e^{-t^{2} / 2} \\
& \quad+\frac{n-1}{2 n} \frac{1}{n^{2}}\left(\frac{\theta_{4}}{4 !}\right)^{2}(i t)^{8} e^{-t^{2} / 2} \\
& \quad+n \mu^{n-1}\left(\gamma \frac{\beta_{6}}{6 !}\left(\frac{t}{\sqrt{n}}\right)^{6}+\gamma \frac{\theta_{8}^{(4)}}{8 !}\left(\frac{t}{\sqrt{n}}\right)^{8}+\gamma \frac{\left\|\theta_{10}^{(4)}\right\|}{10 !}\left(\frac{t}{\sqrt{n}}\right)^{10}\right) \\
& \quad+C_{n}^{2} \mu^{n-1}\left(\gamma \frac{\theta_{4}}{4 !} \frac{\beta_{6}}{6 !}\left(\frac{t}{\sqrt{n}}\right)^{10}+2 \gamma \frac{\theta_{4}}{4 !} \frac{\theta_{6}^{(4)}}{6 !}\left(\frac{t}{\sqrt{n}}\right)^{10}+\gamma \frac{\theta_{4}}{4 !} \frac{\left\|\theta_{8}^{(4)}\right\|}{8 !}\left(\frac{t}{\sqrt{n}}\right)^{12}\right) \\
& \quad+C_{n}^{2} \mu^{n-1} \gamma \frac{\theta_{6}^{(4)}}{6 !}\left(\frac{\beta_{6}}{6 !}+\frac{1}{48}\right)\left(\frac{t}{\sqrt{n}}\right)^{12} \\
& \quad+C_{n}^{3} \mu^{n-1}\left(\gamma\left(\frac{\theta_{4}}{4 !}\right)^{3}\left(\frac{t}{\sqrt{n}}\right)^{12}+\gamma\left(\frac{\theta_{4}}{4 !}\right)^{2}\left(\frac{\beta_{6}}{6 !}+\frac{1}{48}\right)\left(\frac{1}{\sqrt{n}}\right)^{14}\right),
\end{aligned}
$$

где символом $\gamma$ обозначень различные функции, абсолютнье величинь которьих не превосходят единицьи, а $\mu^{n-1}=\mu^{n-1}(t / \sqrt{n})$.

Д о к а з а т е л ь с т в о. Доказательство этой леммы опирается на многократное использование хорошо известного равенства

$$
a^{l}-b^{l}=\sum_{j=0}^{l-1} a^{l-j-1} b^{j}(a-b),
$$


которое справедливо для любого натурального $l$ и любых комплексных чисел $a$ и $b$. Воспользуемся также утверждением предыдущей леммы. Нам будет удобно записывать характеристическую функцию $e^{-t^{2} / 2}$ стандартного нормального закона, предельного в ЦПТ, в том же виде, в каком записывается допредельная характеристическая функция, т.е. мы будем записывать $e^{-t^{2} / 2}$ как $g^{n}(t / \sqrt{n})$, где $g(t)=e^{-t^{2} / 2}$. Мы часто будем опускать аргументы у функций $f, g, \mu$ и некоторых других функций, это не должно привести к недоразумениям, все эти аргументы, если не оговорено противное, суть $t / \sqrt{n}$.

По формуле (13) разность

$$
f^{n}-g^{n}=\sum_{j=0}^{n-1} f^{n-j-1} g^{j}(f-g)=\psi \sum_{j=0}^{n-1} f^{n-j-1} g^{j+1},
$$

где $\psi=e^{t^{2} / 2 n} f(t / \sqrt{n})-1$. Используя представление (12) леммы 1 для $k=2$ и $s=2$, видим, что

$$
\begin{aligned}
f^{n}-g^{n}= & \left(\frac{\theta_{4}}{4 !}\left(\frac{i t}{\sqrt{n}}\right)^{4}+\frac{\theta_{6}^{(4)}}{6 !}\left(\frac{i t}{\sqrt{n}}\right)^{6}+\frac{\theta_{8}^{(4)}}{8 !}\left(\frac{i t}{\sqrt{n}}\right)^{8}\right) \sum_{j=0}^{n-1} f^{n-j-1} g^{j+1} \\
& +\left(\gamma \frac{\beta_{6}}{6 !}\left(\frac{t}{\sqrt{n}}\right)^{6}+\gamma \frac{\left\|\theta_{10}^{(4)}\right\|}{10 !}\left(\frac{t}{\sqrt{n}}\right)^{10}\right) \sum_{j=0}^{n-1} f^{n-j-1} g^{j} .
\end{aligned}
$$

Обратим внимание на то, что в первой сумме по $j$ показатели степеней у функции $g$ суть $j+1$, в то время как во второй сумме они суть $j$. Это связано с тем, что во второй сумме одна функция $g$ используется для того, чтобы подавить экспоненты $e^{t^{2} / 2 n}$, происходящие от экспонент в двух последних слагаемых правой части (12).

Последнее слагаемое в (14) можно записать как

$$
\gamma \frac{\beta_{6}}{6 !}\left(\frac{t}{\sqrt{n}}\right)^{6} n \mu^{n-1}+\gamma \frac{\left\|\theta_{10}^{(4)}\right\|}{10 !}\left(\frac{t}{\sqrt{n}}\right)^{10} n \mu^{n-1} .
$$

В таком виде мы включим его в остаточную часть разложения функции $f^{n}(t / \sqrt{n})$. Отметим, что все функции $\gamma$, абсолютные значения которых не превосходят единицы, в этих выкладках различны.

Рассмотрим величину

$$
\frac{\theta_{8}^{(4)}}{8 !}\left(\frac{i t}{\sqrt{n}}\right)^{8} \sum_{j=0}^{n-1} f^{n-j-1} g^{j+1}
$$

которую можно выделить из правой части (14). Ее можно записать как

$$
\gamma \frac{\theta_{8}^{(4)}}{8 !}\left(\frac{t}{\sqrt{n}}\right)^{8} n \mu^{n-1}
$$


и в таком виде включить в остаточную часть разложения леммы.

Рассмотрим величину

$$
\frac{\theta_{6}^{(4)}}{6 !}\left(\frac{i t}{\sqrt{n}}\right)^{6} \sum_{j=0}^{n-1} f^{n-j-1} g^{j+1}
$$

которую можно выделить из правой части (14). Запишем сумму по $j$ в виде

$$
\sum_{j_{1}=0}^{n-1} f^{n-j_{1}-1} g^{j_{1}+1}=\sum_{j_{1}=0}^{n-2}\left(f^{n-j_{1}-1}-g^{n-j_{1}-1}\right) g^{j_{1}+1}+n g^{n} .
$$

Вновь используя равенство (13), мы видим, что последняя сумма по $j_{1}$ равна

$\sum_{j_{1}=0}^{n-2} \sum_{j_{2}=0}^{n-j_{1}-2} f^{n-j_{1}-j_{2}-2} g^{j_{2}}(f-g) g^{j_{1}+1}=\sum_{0 \leqslant j_{1}+j_{2} \leqslant n-2} f^{n-j_{1}-j_{2}-2} g^{j_{1}+j_{2}+1}(f-g)$.

В правой части последнего равенства суммирование проводится по всем наборам неотрицательных целых чисел $j_{1}, j_{2}$ таких, что их сумма не превосходит $n-2$. Аналогичные переходы от повторных сумм к кратным приходится делать довольно часто, и в связи с этим отметим, что число наборов неотрицательных целых чисел $j_{1}, \ldots, j_{s}, s$ - натуральное, таких, что $j_{1}+\cdots+j_{s} \leqslant n-s$ равно $C_{n}^{s}$.

Из сказанного следует, что величина (15) равна

$$
\frac{\theta_{6}^{(4)}}{6 !}\left(\frac{i t}{\sqrt{n}}\right)^{6} n e^{-t^{2} / 2}+\frac{\theta_{6}^{(4)}}{6 !}\left(\frac{i t}{\sqrt{n}}\right)^{6} \sum_{0 \leqslant j_{1}+j_{2} \leqslant n-2} f^{n-j_{1}-j_{2}-2} g^{j_{1}+j_{2}+1}(f-g) .
$$

Первое из этих слагаемых мы включим в главную часть разложения.

Для оценки суммы оставшихся слагаемых в (16) воспользуемся неравенством

$$
|f-g| \leqslant \frac{\left|\theta_{4}\right|}{4 !}\left(\frac{t}{\sqrt{n}}\right)^{4}+\left(\frac{\beta_{6}}{6 !}+\frac{1}{48}\right)\left(\frac{t}{\sqrt{n}}\right)^{6}
$$

которое легко следует из разложений в отрезки рядов Тейлора

$$
f(t)=1-\frac{t^{2}}{2}+\frac{\alpha_{4}}{4 !} t^{4}+\gamma \frac{\beta_{6}}{6 !} t^{6}, \quad e^{-t^{2} / 2}=1-\frac{t^{2}}{2}+\frac{3}{4 !} t^{4}+\gamma \frac{15}{6 !} t^{6} .
$$

Применяя эту оценку, мы можем сумму оставшихся слагаемых из (16) записать в виде

$$
\gamma \frac{\theta_{4}}{4 !} \frac{\theta_{6}^{(4)}}{6 !}\left(\frac{t}{\sqrt{n}}\right)^{10} C_{n}^{2} \mu^{n-1}+\gamma \frac{\theta_{6}^{(4)}}{6 !}\left(\frac{\beta_{6}}{6 !}+\frac{1}{48}\right)\left(\frac{t}{\sqrt{n}}\right)^{12} C_{n}^{2} \mu^{n-1} .
$$


В таком виде мы и включим ее в остаточную часть разложения.

Рассмотрим величину

$$
\frac{\theta_{4}}{4 !}\left(\frac{i t}{\sqrt{n}}\right)^{4} \sum_{j=0}^{n-1} f^{n-j-1} g^{j+1}
$$

которую можно выделить из (14). Действуя аналогично предыдущему, мы видим, что она равна

$$
\frac{\theta_{4}}{4 !}\left(\frac{i t}{\sqrt{n}}\right)^{4} n e^{-t^{2} / 2}+\frac{\theta_{4}}{4 !}\left(\frac{i t}{\sqrt{n}}\right)^{4} \sum_{0 \leqslant j_{1}+j_{2} \leqslant n-2} f^{n-j_{1}-j_{2}-2} g^{j_{1}+j_{2}+1}(f-g) .
$$

Первое слагаемое мы включим в главную часть разложения леммы.

Преобразуем второе слагаемое, воспользовавшись утверждением леммы 1 при $k=2, s=1$. Мы можем записать его в виде

$$
\begin{aligned}
& \frac{\theta_{4}}{4 !}\left(\frac{i t}{\sqrt{n}}\right)^{4}\left(\frac{\theta_{4}}{4 !}\left(\frac{i t}{\sqrt{n}}\right)^{4}+\frac{\theta_{6}^{(4)}}{6 !}\left(\frac{i t}{\sqrt{n}}\right)^{6}\right) \sum_{0 \leqslant j_{1}+j_{2} \leqslant n-2} f^{n-j_{1}-j_{2}-2} g^{j_{1}+j_{2}+2} \\
& +\frac{\theta_{4}}{4 !}\left(\frac{i t}{\sqrt{n}}\right)^{4}\left(\gamma \frac{\beta_{6}}{6 !}\left(\frac{i t}{\sqrt{n}}\right)^{6}+\gamma \frac{\left\|\theta_{8}^{(4)}\right\|}{8 !}\left(\frac{t}{\sqrt{n}}\right)^{8}\right) \\
& \quad \times \sum_{0 \leqslant j_{1}+j_{2} \leqslant n-2} f^{n-j_{1}-j_{2}-2} g^{j_{1}+j_{2}+1}
\end{aligned}
$$

Второе слагаемое мы можем записать как

$$
\gamma \frac{\theta_{4}}{4 !} \frac{\beta_{6}}{6 !}\left(\frac{t}{\sqrt{n}}\right)^{10} C_{n}^{2} \mu^{n-1}+\gamma \frac{\theta_{4}}{4 !} \frac{\left\|\theta_{8}^{(4)}\right\|}{8 !}\left(\frac{t}{\sqrt{n}}\right)^{12} C_{n}^{2} \mu^{n-1} .
$$

В таком виде мы и включим его в остаточную часть разложения.

Сумму величин из (19), связанную с $\theta_{6}^{(4)}$, можно записать в виде

$$
\gamma \frac{\theta_{4}}{4 !} \frac{\theta_{6}^{(4)}}{6 !}\left(\frac{t}{\sqrt{n}}\right)^{10} C_{n}^{2} \mu^{n-1}
$$

Такая величина уже встречалась нам в (18), правда, функция $\gamma$ там была, вообще говоря, иной, но нетрудно понять, что в итоге мы должны включить в остаточную часть разложения величину

$$
2 \gamma \frac{\theta_{4}}{4 !} \frac{\theta_{6}^{(4)}}{6 !}\left(\frac{t}{\sqrt{n}}\right)^{10} C_{n}^{2} \mu^{n-1}
$$

Нам осталось рассмотреть

$$
\left(\frac{\theta_{4}}{4 !}\right)^{2}\left(\frac{i t}{\sqrt{n}}\right)^{8} \sum_{0 \leqslant j_{1}+j_{2} \leqslant n-2} f^{n-j_{1}-j_{2}-2} g^{j_{1}+j_{2}+2} .
$$


Действуя аналогично предыдущему, легко убедиться в том, что эта величина совпадает с

$$
\begin{aligned}
& C_{n}^{2}\left(\frac{\theta_{4}}{4 !}\right)^{2}\left(\frac{i t}{\sqrt{n}}\right)^{8} e^{-t^{2} / 2} \\
& \quad+\left(\frac{\theta_{4}}{4 !}\right)^{2}\left(\frac{i t}{\sqrt{n}}\right)^{8} \sum_{0 \leqslant j_{1}+j_{2}+j_{3} \leqslant n-3} f^{n-j_{1}-j_{2}-j_{3}-3} g^{j_{1}+j_{2}+j_{3}+2}(f-g) .
\end{aligned}
$$

Первое слагаемое мы отнесем в главную часть разложения леммы.

Если для суммы оставшихся слагаемых в (20) воспользоваться оценкой (17), то мы сможем записать ее в виде

$$
\gamma\left(\frac{\theta_{4}}{4 !}\right)^{3}\left(\frac{t}{\sqrt{n}}\right)^{12} C_{n}^{3} \mu^{n-1}+\gamma\left(\frac{\theta_{4}}{4 !}\right)^{2}\left(\frac{\beta_{6}}{6 !}+\frac{1}{48}\right)\left(\frac{t}{\sqrt{n}}\right)^{14} C_{n}^{3} \mu^{n-1}
$$

Эту сумму мы включаем в остаточную часть разложения леммы.

Собирая вместе полученные результаты, мы убеждаемся в справедливости утверждения леммы.

Лемма 3. Для функиии $f^{n}(t / \sqrt{n})$, где $f$ - характеристическая функиия симметричного распределения с единичной дисперсией и конечным моментом шестого порядка при любом натуральном $n \geqslant 4$ справедливо следующее представление:

$$
\begin{aligned}
& f^{n}\left(\frac{t}{\sqrt{n}}\right)=e^{-t^{2} / 2}+\frac{\theta_{4}}{4 ! n}(i t)^{4} e^{-t^{2} / 2}+\frac{\theta_{6}^{(4)}}{6 ! n^{2}}(i t)^{6} e^{-t^{2} / 2} \\
& +\frac{n-1}{2 n} \frac{1}{n^{2}}\left(\frac{\theta_{4}}{4 !}\right)^{2}(i t)^{8} e^{-t^{2} / 2}+\frac{\theta_{8}^{(4)}}{8 ! n^{3}}(i t)^{8} e^{-t^{2} / 2} \\
& +\frac{n-1}{n} \frac{1}{n^{3}} \frac{\theta_{4}}{4 !} \frac{\theta_{6}^{(4)}}{6 !}(i t)^{10} e^{-t^{2} / 2}+\frac{(n-1)(n-2)}{6 n^{2}} \frac{1}{n^{3}}\left(\frac{\theta_{4}}{4 !}\right)^{3}(i t)^{12} e^{-t^{2} / 2} \\
& +n \mu^{n-1}\left(\gamma \frac{\beta_{6}}{6 !}\left(\frac{t}{\sqrt{n}}\right)^{6}+\gamma \frac{\theta_{10}^{(4)}}{10 !}\left(\frac{t}{\sqrt{n}}\right)^{10}+\gamma \frac{\left\|\theta_{12}^{(4)}\right\|}{12 !}\left(\frac{t}{\sqrt{n}}\right)^{12}\right) \\
& +C_{n}^{2} \mu^{n-1}\left(\gamma \frac{\theta_{4}}{4 !} \frac{\beta_{6}}{6 !}\left(\frac{t}{\sqrt{n}}\right)^{10}+2 \gamma \frac{\theta_{4}}{4 !} \frac{\theta_{8}^{(4)}}{8 !}\left(\frac{t}{\sqrt{n}}\right)^{12}+\gamma\left(\frac{\theta_{6}^{(4)}}{6 !}\right)^{2}\left(\frac{t}{\sqrt{n}}\right)^{12}\right. \\
& \left.+\gamma \frac{\theta_{6}^{(4)}}{6 !} \frac{\beta_{6}}{6 !}\left(\frac{t}{\sqrt{n}}\right)^{12}\right) \\
& +C_{n}^{2} \mu^{n-1}\left(\gamma \frac{\theta_{4}}{4 !} \frac{\left\|\theta_{10}^{(4)}\right\|}{10 !}\left(\frac{t}{\sqrt{n}}\right)^{14}+\gamma \frac{\theta_{6}^{(4)}}{6 !} \frac{\left\|\theta_{8}^{(4)}\right\|}{8 !}\left(\frac{t}{\sqrt{n}}\right)^{14}\right. \\
& \left.+\gamma\left(\frac{\beta_{6}}{6 !}+\frac{1}{48}\right) \frac{\theta_{8}^{(4)}}{8 !}\left(\frac{t}{\sqrt{n}}\right)^{14}\right) \\
& +C_{n}^{3} \mu^{n-1}\left(3 \gamma\left(\frac{\theta_{4}}{4 !}\right)^{2} \frac{\theta_{6}^{(4)}}{6 !}\left(\frac{t}{\sqrt{n}}\right)^{14}+\gamma\left(\frac{\theta_{4}}{4 !}\right)^{2} \frac{\beta_{6}}{6 !}\left(\frac{t}{\sqrt{n}}\right)^{14}\right.
\end{aligned}
$$




$$
\begin{gathered}
\left.+2 \gamma \frac{\theta_{4}}{4 !} \frac{\theta_{6}^{(4)}}{6 !}\left(\frac{\beta_{6}}{6 !}+\frac{1}{48}\right)\left(\frac{t}{\sqrt{n}}\right)^{16}\right) \\
+C_{n}^{3} \mu^{n-1} \gamma\left(\frac{\theta_{4}}{4 !}\right)^{2} \frac{\left\|\theta_{8}^{(4)}\right\|}{8 !}\left(\frac{t}{\sqrt{n}}\right)^{16} \\
+C_{n}^{4} \mu^{n-1}\left(\gamma\left(\frac{\theta_{4}}{4 !}\right)^{4}\left(\frac{t}{\sqrt{n}}\right)^{16}+\gamma\left(\frac{\theta_{4}}{4 !}\right)^{3}\left(\frac{\beta_{6}}{6 !}+\frac{1}{48}\right)\left(\frac{t}{\sqrt{n}}\right)^{18}\right),
\end{gathered}
$$

где символом $\gamma$ обозначень различнье функиии, абсолютные величинь которьх не превосходят единицьи, а $\mu^{n-1}=\mu^{n-1}(t / \sqrt{n})$.

Доказательство этой леммы аналогично доказательству леммы 2 , привлечения новых идей не требуется, поэтому мы его опускаем.

\section{СПИСОК ЛИТЕРАТУРЫ}

1. Гамкрелидзе Н. Г. Муавра-Лапласа теорема. Вероятность и математическая статистика. Энциклопедия. М.: Большая российская энциклопедия, 1999.

2. Петров В. В. Суммы независимых случайных величин. М.: Наука, 1972, 416 с.

3. Прохоров Ю. В., Розанов Ю.А. Теория вероятностей. Основные понятия. Предельные теоремы. Случайные процессы. 2 изд., М.: Наука, 1973.

4. Сенатов B. В. Центральная предельная теорема: Точность аппроксимации и асимптотические разложения. М.: Книжный дом «Либроком», 2009, 352 с.

5. Сенатов В.В. О неулучшаемых оценках для асимптотических разложений в ЦПТ. - Теория вероятн. и ее примен., 2012, т. 57, в. 4, с. 640-666.

6. Шевцова И. Г. Об асимптотически правильных постоянных в неравенстве БерриЭссеена. - Теория вероятн. и ее примен., 2010, т. 55, в. 3, с. 619-621.

Поступила в редакцию 27.II.2012

Сокращенный вариант 15.IV.2015 